\title{
Duty Hour Reform and Internal Medicine Residency Training: No Time to Lose
}

\author{
Diane B. Wayne, $M D^{7}$ and Vineet M. Arora, MD, MAPP ${ }^{2}$ \\ 'Department of Medicine, Northwestern University Feinberg School of Medicine, Chicago, IL, USA; ${ }^{2}$ Department of Medicine, University of \\ Chicago Pritzker School of Medicine, Chicago, IL, USA.
}

$\mathrm{J}$ Gen Intern Med 24(10):1169-70

DOI: $10.1007 / \mathrm{s} 11606-009-1093-7$

(c) Society of General Internal Medicine 2009

I $\mathrm{n}$ the six years since restrictions were placed on resident work hours, much discussion and debate have ensued regarding the impact of these limitations on resident education and patient care. In 2008, the Institute of Medicine released a report encouraging further limits on resident work hours including a maximum shift of 16 hours unless five hours of protected sleep was provided. ${ }^{1}$ In response to this, the Accreditation Council for Graduate Medical Education (ACGME) is currently reviewing resident duty hours with a pledge to "completely examine resident duty hour standards, with a goal of making them better." ${ }^{2}$ Although further duty hour limits for residents seem likely, there is no consensus on the impact of the initial regulations on patient care and education. In light of this, we believe internal medicine residency educators must actively participate in these discussions and serve as advocates for patients and residents through innovation and research.

In this issue of JGIM, Volpp and colleagues advance what is known about the impact of duty hour reform on the quality of patient care. ${ }^{3}$ This work represents the latest in an important series of papers this group has written on the subject. In 2007, initial analyses showed that duty hour limitations did not lead to increased mortality for patients and may have had slight benefits for certain subgroups. ${ }^{4,5}$ However, subsequent reports have not demonstrated clear benefits for patients. These include a study documenting that duty hour reform had no systematic impact on rates of ten procedural and surgical patient safety indicators. ${ }^{6}$

In the current paper, Volpp et al. evaluate the impact of duty hour reform on outcomes among the highest-risk patients. Their hypothesis was that higher severity patients might be more vulnerable to benefit or harm from changes in health care delivery such as duty hour limitations. To assess this, they performed an observational study of almost nine million Medicare recipients and VA patients using interrupted time series analysis of data from July 1, 2000 to June 30, 2005.

Dr. Arora receives grant funding from the Accreditation Council of Graduate Medical Education (ACGME) and honoraria from the American Board of Internal Medicine. She has provided testimony on resident duty hours to the Institute of Medicine and to the ACGME Duty Hours Congress as a representative of the American College of Physicians. Published online August 28, 2009
Outcomes assessed were 30-day mortality in high acuity patients and deaths in patients who suffered a post-operative complication. The researchers found that 30-day mortality and death after post-operative complications were overall unchanged in the pre- and post-duty hour reform periods. Although some improvement was seen in VA surgical patients in post-reform year 1 , the authors conclude that duty hour reform had no overall benefit to the outcomes of high risk medical and surgical hospitalized patients. As no consistent benefit has been shown in this and other studies, we agree with others who have called for more research before implementing further restrictions in resident duty hours in an attempt to improve patient care. ${ }^{7}$

This current study continues the high quality work of Volpp and colleagues to systematically evaluate the impact of duty hour reform on patient care using administrative data. Strengths of the study include the large study sample and evaluation of patients at VA Medical Centers as well as non federal acute-care hospitals. However, the study does have some important limitations. As residency educators know, many changes were made in hospitals in response to reduced resident hours. These changes, such as increased direct patient care by faculty members and fellows, and the expansion of non-teaching hospitalist services, may have offset any impact of duty hour reform on the mortality rates of high-risk patients. Without understanding and accounting for these changes, it is difficult to isolate the effect of shortening resident hours on patient care. Another limitation is that the study ended in 2005, only two years after the initiation of ACGME duty hour requirements, when compliance was suboptimal. ${ }^{8}$ The true impact of duty hour reform might require more time to become apparent after programs achieved substantially higher compliance rates. Finally, the impact of duty hour reform on outcomes other than mortality, such as readmission rates, cost or decreased length of stay remains uncertain.

In considering how to optimize duty hours, patient safety and resident education, it is important to consider how duty hour restrictions might make patients safer. The proposed rationale is based on research that demonstrates that fatigued clinicians make more errors, which presumably result in patient harm or death. Therefore, it follows that a reduction in work hours might lead to well rested residents who make fewer mistakes. thus leading to improved patient survival. In thinking about an average internal medicine resident, this hypothesis requires several assumptions to hold true. First, residents must use additional time gained from working less to get more sleep. Second, the contribution of fatigue to patient mortality has to be greater than the combination of increased handoffs and greater work intensity. There are several reasons 
why these assumptions may not hold true. First, while a reduction in work hours likely leads to a modest increase in sleep, a variety of competing responsibilities may prevent internal medicine residents from receiving adequate preventive and recovery sleep to guard against fatigue. ${ }^{9}$ Second, patient deaths in teaching hospitals are likely multifactorial, and resident fatigue may not consistently be the most important contributor. Even the death of Libby Zion, which ultimately heralded resident duty hour restrictions in New York 20 years ago, was attributed to a multitude of factors, such as inadequate supervision, workload, and fatigue. ${ }^{10}$

Another barrier to improved patient care through duty hour restriction is that the risk of mistakes by a fatigued resident may be counterbalanced by the risk of a handoff to a well rested resident who is not as familiar with the patient. In thinking about this balance, it is important to remember that not all resident work is the same. There may be some tasks that are more susceptible to errors due to fatigue and others that are more prone to errors due to handoffs. Numerous studies suggest that tasks that require a high degree of vigilance, such as inserting an arterial line or observing a monitor during anesthesia, are most susceptible to fatigue. ${ }^{11}$ In contrast, there are other tasks that require a priori knowledge of the patient (i.e. interpreting mental status changes in a patient with baseline dementia or leading a family discussion for a patient who is dying) that may be more prone to errors during handoffs. While improvements in handoffs are certainly needed, it is not known whether these improvements can substitute for continuity of care.

In light of these uncertainties, it remains unclear how internal medicine residency programs should respond to potential future work hour limitations. Is it better for internal medicine resident education and patient safety to limit shifts to 16 hours or provide five hours of protected sleep during overnight call? How important is preserving overnight call, or providing the opportunity to follow newly admitted patients through their initial presentation, to the education and experience of internal medicine residents? How should we balance further duty hour limitations against concerns regarding additional handoffs and professionalism? Finally, internal medicine residency educators need to consider the overall content and delivery of education in the era of duty hour reform. Can fewer hours in direct patient care be enhanced by simulated patient care or e-learning? What are the best methods to rigorously assess the development of competency in residents? Is it possible that skills could actually improve despite fewer hours engaged in direct patient care? How can we ensure that residents spend their time on education and clinical care as opposed to administrative tasks that can be performed by non-physician personnel? At a time of great economic strain for teaching hospitals, what are the most cost effective strategies to improve patient safety and resident education?

Residency training is a period of rapid personal and professional growth, and internal medicine residency educators must strive to preserve a high quality experience for patients and trainees. Unfortunately, the first six years of duty hour reform have left us with more questions than answers about how to best educate residents and provide optimal patient care. As Volpp and colleagues have shown us, there are no easy answers to these questions and we still have a great deal of work to do. While more research is needed, rigorous research into each of these questions may take years to produce and disseminate. In the interim, internal medicine residency educators must continue to innovate and advocate for solutions that maximize patient safety and resident education. Given the possibility of future duty hour restrictions, there is no time to lose.

Corresponding Author: Diane B. Wayne, MD, Department of Medicine, Northwestern University Feinberg School of Medicine, 251 E. Huron St., Galter 3-150, Chicago, IL 60611, USA (e-mail: dwayne@northwestern.edu).

\section{REFERENCES}

1. Institute of Medicine. Optimizing graduate medical trainee (resident) hours and work schedules to improve patient safety. 2007.

2. Open Letter to GME Community from Thomas Nasca, MD, MACP, ACGME CEO. Available at: http://www.acgme.org/acWebsite/home/ nascaletter_feb2009.pdf. Accessed on August 2, 2009.

3. Volpp KG, Rosen AK, Rosenbaum PR, et al. Did duty hour reform lead to better outcomes among the highest risk patients? J Gen Intern Med. 2009. doi:10.1007/s11606-009-1011-z

4. Volpp KG, Rosen AK, Rosenbaum PR, et al. Mortality among hospitalized Medicare beneficiaries in the first two years following ACGME resident duty hour reform. JAMA. 2007;298:975-83.

5. Volpp KG, Rosen AK, Rosenbaum PR, et al. Mortality among patients in VA hospitals in the first two years following ACGME resident duty hour reform. JAMA. 2007;298:984-92.

6. Rosen AK, Loveland SA, Romano PS, et al. Effects of resident duty hour reform on patient safety among hospitalized Veterans Health Adminstration and Medicare patients. Med Care. 2009;47:723-31.

7. Blanchard MS, Meltzer D, Polonsky KS. To nap or not to nap? Residents' work hours revisited. NEJM. 2009;360:2242-4.

8. Landrigan CP, Barger LK, Cade BE, Ayas NT, Czeisler CA. Interns' compliance with accreditation council for graduate medical education work-hour limits. JAMA. 2006;296(9):1063-70.

9. Arora VM, Georgitis E, Woodruff JN, Humphrey HJ, Meltzer D. Improving sleep hygiene of medical interns: can the sleep, alertness, and fatigue education in residency program help? Arch Intern Med. 2007; 167(16): 1738-44.

10. Bell BM. Supervision, not regulation of hours, is the key to improving the quality of patient care. JAMA. 1993;269:403-4.

11. Weinger MB. Vigilance, boredom, and sleepiness. J Clin Monit Comput. 1999;15:549-52. 